\title{
Punção liquórica cervical lateral em C1-C2: um procedimento de grande utilidade em pacientes vítimas de trauma - Relato de série de casos
}

\section{Lateral Cervical Spinal Puncture at c1-c2: A Useful Procedure in Trauma Patients - Report of a Series of}

\section{Cases}

\author{
Rodrigo Moreira Faleiro ${ }^{1}$ Sérgio Augusto Vieira Cançado ${ }^{2}$ Luiz Alberto Otoni Garcia ${ }^{2}$ \\ Paula de Castro Menezes Cândido ${ }^{3}$ Luanna Rocha Vieira Martins ${ }^{4}$
}

${ }^{1}$ Chefe do serviço de Neurocirurgia e Neurologia do Hospital João XXIII;

Neurocirurgião do Hospital Felício Rocho, Belo Horizonte, MG, Brasil

2 Residente de Neurocirurgia da Fundação Hospitalar do Estado de

Minas Gerais (FHEMIG), Belo Horizonte, MG, Brasil

${ }^{3}$ Residente de Radiologia do Hospital Felício Rocho, Belo Horizonte, MG, Brasil

${ }^{4}$ Acadêmica de medicina da Universidade Federal de Minas Gerais (UFMG), Belo Horizonte, MG, Brasil

Arq Bras Neurocir 2015;34:30-33.

\begin{abstract}
Address for correspondence Rodrigo Moreira Faleiro, MSc, Rua Caraça, 518/201, Serra, Belo Horizonte, Minas Gerais, Brasil, CEP 30220-260 (e-mail: r.m.faleiro@hotmail.com).

Hospital João XXIII da Fundação Hospitalar do Estado de Minas Gerais (FHEMIG), Belo Horizonte, MG, Brasil
\end{abstract}

\section{Resumo}

\section{Palavras-chave}

- punção espinal

- pressão do líquido cefalorraquidiano

- ferimentos e lesões
A punção liquórica cervical lateral no espaço C1-C2 foi descrita a partir de uma modificação da técnica utilizada para cordotomia percutânea anterolateral. Neste artigo, é descrita uma série de 14 pacientes vítimas de trauma, submetidos à punção liquórica cervical lateral. Todos os procedimentos foram realizados para pesquisa de meningite bacteriana. Presença de escara lombar e occipital, fratura instável de coluna vertebral, tração ou imobilização ortopédica e ventilação mecânica controlada foram os principais motivos para se optar pela punção liquórica cervical lateral. A taxa de sucesso foi de $93 \%$. Não foram evidenciadas maiores complicações per ou pósprocedimento. A punção liquórica cervical lateral se mostrou um método eficaz para obtenção de líquor em pacientes vítimas de trauma, pois não requer mudança de decúbito ou angulação da cabeceira, o que evita complicações em pacientes entubados e com fratura instável de coluna vertebral. Estudos prévios sugerem que a punção liquórica cervical lateral deve ser o método de escolha em pacientes vítimas de trauma com restrição de mobilização.

The lateral cervical spinal puncture at $\mathrm{C} 1-\mathrm{C} 2$ was described from a modification of the technique used for percutaneous anterolateral cordotomy. In this article, we describe a series of 14 patients of multiple trauma victims who underwent lateral cervical spinal received

February 19, 2014

accepted

November 1, 2014
DOI http://dx.doi.org/ $10.1055 / \mathrm{s}-0035-1547383$ ISSN $0103-5355$.
Copyright $(2015$ by Thieme Publicações License terms Ltda, Rio de Janeiro, Brazil
$(\Theta(1) \Theta \circledast$ 


\section{Keywords}

- spinal puncture

- cerebrospinal fluid pressure

- wounds and injuries puncture. All procedures were performed as a screening for bacterial meningitis. Low back and occipital ulcer, unstable spinal fractures, orthopedic traction or immobilization and ventilated patients were the main reasons to opt for the lateral cervical spinal puncture over standard techniques of liquor puncture. The success rate was $93 \%$. There was not major complication per or post procedural. The lateral cervical spinal puncture proved to be an effective method of obtaining liquor in victims of trauma because it does not requires changing position or rotation of the head, preventing complications in patients with orotraqueal tube and unstable spinal fractures. Previous studies suggest that the lateral cervical spinal puncture should be the method of choice in trauma patients with restriction of mobilization.

\section{Introdução}

A punção cervical lateral (PCL), realizada no espaço entre C1$\mathrm{C} 2$, foi inicialmente empregada na cordotomia anterolateral cervical percutânea, em que fibras espinotalâmicas eram interrompidas para alívio de dor de difícil tratamento clínico. ${ }^{1}$ A seguir, foi utilizada para realização de mielografia com contraste, ${ }^{2}$ para mielografia gasosa ${ }^{3}$ e, por fim, para obtenção de líquor. ${ }^{4,5}$ Nos últimos anos, com o advento da ressonância nuclear magnética, esta técnica vem sendo cada vez menos utilizada.

A PCL constitui método alternativo a outras modalidades de punção liquórica, como suboccipital mediana e lombar. Esta técnica (PCL) se torna bem indicada para pacientes traqueostomizados, monitorados, sob respiração assistida, assim como em vítimas de trauma com fraturas em que há contraindicação formal para mobilização no leito. ${ }^{4}$ Nestas situações, há dificuldades técnicas com o emprego das formas padrões de punção liquórica.

As contraindicações para realização da PCL são as mesmas que para a punção lombar e suboccipital, ou seja, infecção local, lesão óssea local, hipertensão intracraniana com risco iminente de herniação, entre outras. ${ }^{6}$

As possíveis complicações da PCL são lesões de estruturas nervosas e vasculares (como punção inadvertida da artéria vertebral extracraniana), precipitação de hérnias em pacientes com hipertensão intracraniana, infecções e cefaleia pós-punção. ${ }^{7}$

A PCL é um método simples, seguro e eficaz para obtenção de líquor em pacientes vítimas de trauma, visto que não requer mudança de decúbito ou da angulação da cabeça, o que evita complicações em pacientes com comprometimento pulmonar e fratura instável de coluna vertebral.

A seguir, é descrita uma série de casos de pacientes vítimas de trauma em que foi realizada a PCL.

\section{Relato de Casos}

Foram avaliados 14 pacientes internados no Hospital ProntoSocorro João XXIII no ano de 2013, vítimas de trauma e submetidos à punção cervical lateral (PCL). Grande parte dos pacientes tinha traumatismo cranioencefálico (TCE) grave à admissão. Os demais foram admitidos com diagnóstico de acidente vascular encefálico (AVE) hemorrágico, controle de meningite pós-traumática e pós-operatório de hematoma extradural agudo.

Os aspectos analisados em relação aos pacientes e a esta técnica de punção liquórica foram: idade, diagnóstico, número de tentativas para obtenção de líquor, lado puncionado, resultado (obtenção de sucesso ou não) e justificativa para realização da PCL (- Tabela $\mathbf{1}$ ).

Foram realizados 15 procedimentos, sendo 3 à direita e 12 à esquerda. Em um paciente da série, foram realizados dois procedimentos em momentos distintos. Todos foram realizados com o objetivo de pesquisar presença de meningite bacteriana.

Presença de escara lombar e occipital em 7\% dos casos; fratura instável de coluna vertebral, tração ou imobilização ortopédica em $14 \%$; pneumonia e contusão pulmonar grave (ventilação mecânica) em $73 \%$ e outras condições em $8 \%$ dos casos foram as razões para se optar pela PCL em detrimento das técnicas convencionais de punção liquórica.

A taxa de sucesso foi de $93 \%$. Em apenas um paciente do sexo masculino, de 76 anos e com AVE hemorrágico, não foi obtido sucesso. Não foi evidenciada complicação maior ou menor per ou pós-procedimento, sendo que o nível de consciência e sedação dos pacientes pode ter subestimado complicações sutis.

\section{Discussão}

A PCL constitui método alternativo a outras técnicas de punção liquórica, como lombar e suboccipital mediana. No passado, por volta da década de 1980 , foi muito utilizada para procedimentos neurorradiológicos. ${ }^{7}$ Com o advento da ressonância nuclear magnética, seu uso com essa finalidade perdeu importância.

Recentemente, a PCL, uma técnica quase esquecida, teve sua relevância retomada no cenário dos pacientes vítimas de trauma, o que pode ser exemplificado pelo seu uso cada vez mais frequente em nosso serviço no Hospital Pronto-Socorro João XXIII. A PCL não requer mobilização no leito ou angulação da cabeceira, o que evita complicações em pacientes traumatizados, em especial aqueles com fratura instável de coluna vertebral. A PCLé um ótimo método de escolha nesses pacientes, em especial aqueles com indicação de mobilização 
32 Punção liquórica cervical lateral no paciente vítima de trauma Faleiro et al.

Tabela 1 Tabela descritiva dos pacientes da série

\begin{tabular}{|l|l|l|l|l|l|l|}
\hline Paciente & Idade & Diagnóstico & Tentativas & Lado & Sucesso & Justificativa \\
\hline $1^{*}$ & 67 & TCE grave fechado & 1 & Esquerdo & Sim & Ventilação mecânica \\
\hline 2 & 40 & $\begin{array}{l}\text { Controle de tratamento } \\
\text { de meningite }\end{array}$ & 1 & Esquerdo & Sim & Ventilação mecânica \\
\hline 3 & 35 & TCE grave fechado & 1 & Direito & Sim & Ventilação mecânica \\
\hline 4 & 34 & TCE grave fechado & 5 & Direito & Sim & Ventilação mecânica \\
\hline 5 & 76 & AVE hemorrágico & 8 & Esquerda & Não & Ventilação mecânica \\
\hline 6 & 57 & TCE grave fechado & 1 & Direita & Sim & Ventilação mecânica \\
\hline 7 & 16 & TCE grave fechado & 2 & Esquerda & Sim & Ventilação mecânica \\
\hline 8 & 33 & $\begin{array}{l}\text { Hematoma extradural } \\
\text { agudo pós-operatório }\end{array}$ & 2 & Esquerdo & Sim & Ventilação mecânica \\
\hline 9 & 18 & TCE grave por PAF & 1 & Esquerdo & Sim & Ventilação mecânica \\
\hline 10 & 22 & TCE grave fechado & 4 & Esquerdo & & Fratura ortopédica com tração \\
\hline 11 & 26 & TCE grave por PAF & 1 & Esquerdo & Sim & Escara lombar e occipital \\
\hline 12 & 44 & TCE grave fechado & 1 & Esquerdo & Sim & Ventilação mecânica \\
\hline 13 & 16 & $\begin{array}{l}\text { TCE grave fechado } \\
+ \text { Fístula liquórica }\end{array}$ & 1 & Esquerdo & Sim & Fratura ortopédica com tração \\
\hline 14 & 21 & TCE grave por PAF & 1 & Esquerdo & Sim & Outros \\
\hline
\end{tabular}

*Neste paciente, foram realizados dois procedimentos em momentos distintos.

Abreviações: AVE, acidente vascular encefálico; PAF, projétil por arma de fogo; TCE, traumatismo cranioencefálico.

em bloco e contraindicação de redução da elevação da cabeceira.

Quanto à descrição anatômica do procedimento, as estruturas contidas no trajeto da agulha são: pele e tecido celular subcutâneo, ligamento cervical posterior, plano muscular (oblíquo inferior da cabeça), ligamento atlantoaxial posterior, espaço peridural contendo o plexo venoso vertebral interno e tecido adiposo, e dura-máter ${ }^{6}$ ( - Fig. 1). 0 trajeto da artéria vertebral é caracteristicamente regular, com poucas variações, o que permite que seja respeitada na PCL, a despeito de sua proximidade. A punção da artéria vertebral extracraniana não traz maiores consequências se a agulha for retirada com cuidado; contudo, sua laceração pode provocar espasmo, trombose, hematoma local e fístula arteriovenosa. ${ }^{6}$ Efeitos deletérios da penetração de medula espinhal por agulha relativamente fina são pouco definidos. Contudo, estudos relatam que a penetração acidental da medula espinhal pode causar o sinal de Lhermitte, que consiste em sensações semelhantes a choques elétricos com irradiação comumente descendente pelo tronco e membros. ${ }^{7}$

Quanto à técnica para realização do procedimento, o paciente deve ser posicionado com a cabeça e o pescoço retificados. Os pontos de referência para a inserção situam-se $1 \mathrm{~cm}$ caudal e $1 \mathrm{~cm}$ dorsal à ponta da mastoide, observandose sempre o plano horizontal anatômico. Após realização do

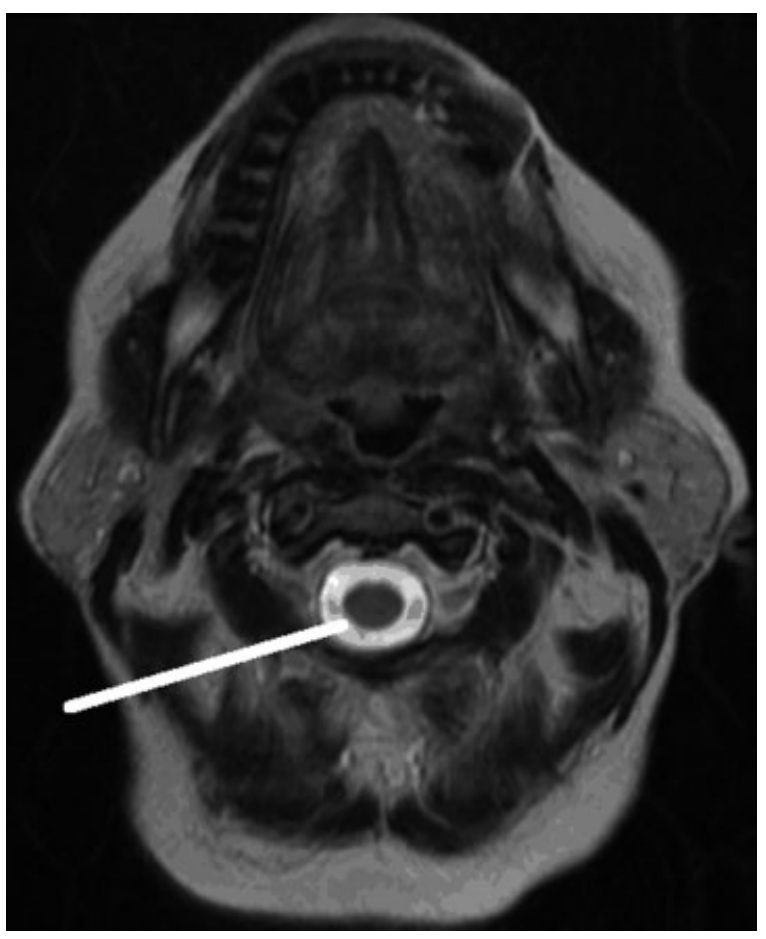

Fig. 1 Tomografia computadorizada do crânio com visualização do trajeto atravessado pela agulha na PCL (traço branco). 


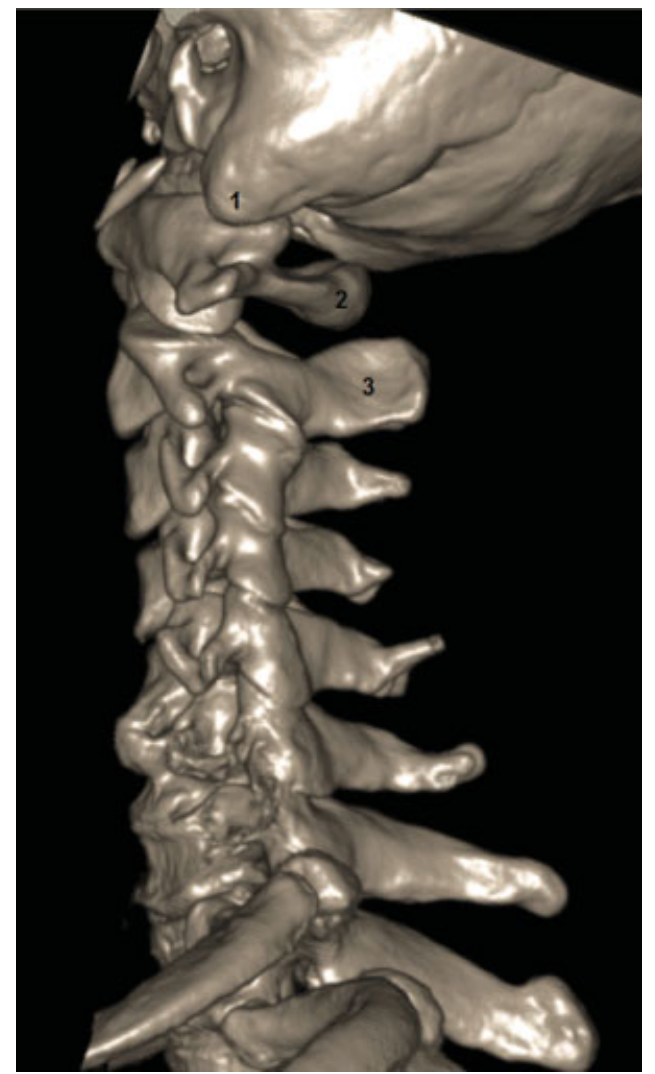

Fig. 2 Punção cervical lateral: pontos de referência para inserção da agulha: (1) mastoide; (2) C1-atlas; (3) C2-áxis.

procedimento, recomenda-se repouso de 24 horas para profilaxia da cefaleia pós-punção. Controle radioscópico durante a inserção da agulha para coleta de líquor pode ser dispensado, sendo que o procedimento pode ser realizado em enfermaria ou no ambulatório, sem desconforto adicional para o paciente ${ }^{6}$ (-Fig. 2).

Conclui-se, portanto, que a PCL é útil em pacientes gravemente enfermos em centros de tratamento intensivo, geralmente em ventilação mecânica ou com restrição de mobilização decorrente de fraturas. Trata-se de técnica simples, segura e com mínimas complicações.

Conflitos de Interesse

Os autores declaram não haver conflitos de interesse.

\section{Referências}

1 Mullan S, Harper PV, Hekmatpanah J, Torres H, Dobbin G. Percutaneous interruption of spinal-pain tracts by means of a strontium 90 needle. J Neurosurg 1963;20:931-939

2 Kelly DL Jr, Alexander E Jr. Lateral cervical puncture for myelography. Technical note. J Neurosurg 1968;29(1):106-110

3 Heinz ER, Goldman RL. The role of gas myelography in neuroradiologic diagnosis. Comments on a new and simple technique. Radiology 1972;102(3):629-634

4 Travassos F, Guedes T, Travassos P. Lateral cervical puncture as a new method for collecting cerebrospinal fluid. Experience in Recife and its metropolitan area. Arq Neuropsiquiatr 1988; 46(1):88-89

5 Zivin JA. Lateral cervical puncture: an alternative to lumbar puncture. Neurology 1978;28(6):616-618

6 Rossitti SL, Balbo RJ. Lateral cervical puncture for myelography and cerebrospinal fluid collection: technical note. Arq Neuropsiquiatr 1988;46(4):397-400

7 Rossitti SL, Balbo RJ. Lhermitte's sign during lateral cervical puncture: survey of possible accidents in the lateral C1-C2 puncture and report of 2 cases of spinal cord penetration]. Arq Neuropsiquiatr 1990;48(3):341-347 